Pacific Journal of Mathematic 


\section{ENDOMORPHISM RINGS OF SELF-GENERATORS}

\section{Birge ZimmermanN-Huisgen}

The group of $R$-homomorphisms $\operatorname{Hom}_{R}(M, A)$, where $M, A$ are modules over a ring $R$, is, in a natural way, a module over the endomorphism ring $S$ of $M$. Under certain weak assumptions on $M$, the following is true: $\operatorname{Hom}_{R}(M,-)$ carries injective envelopes of $R$-modules into injective envelopes of $S$-modules iff $M$ generates all its submodules. Modules of the latter type are called self-generators. For $M$ a selfgenerator, $\operatorname{Hom}_{R}(M,-)$ has additional properties concerning chain conditions and the socle. Many of the known results in this area, in particular those for $M$ projective, are special cases of our main theorems.

Introduction. The question of how properties of a unitary right $R$-module $M=M_{R}$ are related to properties of its endomorphism ring $S$ has been answered completely by the Morita theorems in case $M$ is a progenerator. Then the functors $F=\operatorname{Hom}_{R}(M,-): \mathfrak{M}_{R} \rightarrow \mathfrak{M}_{S}$ and $H=M \otimes_{R}-:{ }_{R} \mathfrak{M} \rightarrow{ }_{S} \mathfrak{M}$ are equivalences and hence preserve and reflect all categorical properties of objects $\left(\mathfrak{M}_{R}\right.$ denotes the category of unitary right $R$-modules).

Anderson [1] determined the finitely generated and projective modules $M$, for which $H$ preserves injective envelopes and called them perfect injectors. Inspired by his paper, we investigate the analogous problem for $F$ and introduce the notion of a "perfect coinjector" along the model of [1] (without restrictions on $M$ ). When $R$ is a Dedekind domain, we have a structure theorem for perfect coinjectors (2.1). It yields a characterization of torsion modules flat over their endomorphism ring which generalizes that for $R=Z$ in [13, Th. 2]. In particular, the perfect coinjectors coincide with those modules generating all their submodules (self-generators) fors for the special choice of $R$. This is false for arbitrary $R$, but it is true (2.4) if certain assumptions, weaker than either "projective" or "generator", are made on ' $M$ (e.g., $M=M T$ where $T$ is the trace ideal of $M$ ).

Large classes of self-generators (§3) justify a closer look: The lattices of $R$-submodules of $A \in \mathfrak{M}_{R}$ and $S$-submodules of Hom $(M, A)$ are intimately related, and so, as a consequence, are the chain conditions and Goldie dimension of $A$ and $\operatorname{Hom}(M, A)$. These correspondences arise as a natural continuation of Sandomierski's results in [15]. Moreover, the self-generators $M=M T$ are exactly those modules, for which $F$ preserves the properties "simple" and "essential" just as in the optimal case, i.e. $M$ a vector space (resulting socleformula: 4.5). 
Another application of $\S 2$ clarifies Anderson's characterization of perfect injectors by means of an equivalence of categories ( $\$ 5)$. At one and the same time, the main result extends results of [1] and supplies additional information about the functor $H$.

This paper is part of the author's doctoral dissertation which was written under the direction of Professor Fr. Kasch at the LudwigMaximilians Universität of Munich. The author wishes to express her gratitude to Professor Fr. Kasch and Professor B. J. Mueller for stimulating conversations and to the referee for his helpful suggestions.

1. The full subcategory $\mathscr{C}$ of $M$-generated objects of $\mathfrak{M}_{R}$. A reference for standard notions and results is [2]. The following notation is observed: $R$ is an associative (not necessarily commutative) ring with $1, \mathfrak{M}_{R}$ the category of unitary right $R$-modules, $M=M_{R}$ an object of $\mathfrak{M}_{R}, S=\operatorname{Hom}_{R}(M, M)$ the ring of $R$-endomorphisms of $M, M^{*}=\operatorname{Hom}_{R}(M, R)$. Naturally, $M$ is a left, $M^{*}$ a right $S$-module.

The homomorphisms (, ): $M^{*} \otimes_{S} M \rightarrow R$ with $(f, m)=f(m)$ resp. [ , ]: $M \otimes_{R} M^{*} \rightarrow S$ with $[m, f]=m f(-)$ are $R$ - $R$-resp. $S$-S-linear, their images are denoted by $T$ resp. $\Delta$. As is well-known, $T=R(\Delta=S)$ means that $M_{R}$ is a generator (finitely generated and projective). $T_{M}(A)=\Sigma\left\{\operatorname{Im}(f), f \in \operatorname{Hom}_{R}(M, A)\right\}$ is called the trace of $M$ on $A$, $T_{M}(R)=T$ is called simply the trace. $\Phi: G F \rightarrow 1_{\mathfrak{R}_{R}}$ represents the natural transformation corresponding to the adjoined pair $(G, F)$, where

$$
\begin{aligned}
& F: \mathfrak{M}_{R} \longrightarrow \mathfrak{M}_{S} \quad \text { with } \quad F(A)=\operatorname{Hom}_{R}(M, A) \\
& G: \mathfrak{M}_{S} \longrightarrow \mathfrak{M}_{R} \quad \text { with } \quad G(B)=B \bigotimes_{S} M
\end{aligned}
$$

One observes $\operatorname{Im}(\Phi(A))=T_{M}(A)$.

It is known that $F$ preserves injective envelopes in case $F$ is full and faithful and $G$ is exact, the latter being true iff $M_{R}$ is a generator [5] (In this statement $\mathfrak{M}_{R}$ may be replaced by any Grothendieck category). Noting that $\operatorname{Hom}(M, A)=\operatorname{Hom}\left(M, T_{M}(A)\right)$, we focus attention on the full subcategory $\mathscr{M}$ of $M$-generated objects of $\mathfrak{M}_{R}$, i.e. $A \in \mathscr{C}$ iff $T_{M}(A)=A$ (compare [4]), with the restricted functors $F^{\prime}: \mathscr{C} \rightarrow \mathfrak{M}_{s}, G^{\prime}: \mathfrak{M}_{S} \rightarrow \mathscr{M}\left(\Phi^{\prime}: G^{\prime} F^{\prime \prime} \rightarrow 1_{\mathscr{M}}\right.$ belongs to the adjoined pair $\left.\left(G^{\prime}, F^{\prime}\right)\right)$. As is easily checked, an injective envelope $A \rightarrow B$ of $R$ modules goes down to an injective envelope $T_{M}(A) \rightarrow T_{M}(B)$ in $\mathscr{M}$, hence $F$ preserves injective envelopes if $F^{\prime}$ does. A sufficient condition for the latter: $F^{\prime \prime}$ full and faithful, $G^{\prime}$ exact. We will interpret this in terms of equivalent conditions on $M$, and we will see that, in many cases, it is also necessary.

Definition 1.1. 1. $M$ is called a self-generator iff $T_{M}(K)=K$, 
for all $R$-submodules $K$ of $M$.

2. $M$ is called a $\Sigma$-self-generator iff $T_{M}(U)=U$, for all $R$-submodules $U$ of $M^{n}, n \in N$. (compare with related concepts in [4] and [10]).

Example 1.2. (F. Dischinger): Let

$$
R=\left\{\left[\begin{array}{llll}
a & 0 & b & 0 \\
0 & a & 0 & c \\
0 & 0 & a & 0 \\
0 & 0 & 0 & a
\end{array}\right]: a, b, c \in K\right\},
$$

where $K$ is a noncommutative field. Choose $\lambda, \mu \in K$ such that $\lambda \mu \neq \mu \lambda$ and let

$$
I=\left\{\left[\begin{array}{cccc}
0 & 0 & b & 0 \\
0 & 0 & 0 & \lambda b \\
0 & 0 & 0 & 0 \\
0 & 0 & 0 & 0
\end{array}\right]: b \in K\right\}
$$

Clearly, $I$ is a right ideal of $R$, and the cyclic right $R$-module $M=$ $R / I$ is a selfgenerator, but not a generator. Denoting the product of $\mu$ with the unity matrix by $x$, we obtain $\mathrm{I} \cap x I=0$ and $R / I \cong$ $R / x I$. Thus $R$ is embedded into $M^{2}$, and consequently $M$ is not $a$ $\Sigma$-self-generator.

Over a commutative ring, clearly, every cyclic module is a $\Sigma$-selfgenerator; for further examples see $\S \S 2,3$. In view of the following two lemmas, Definition 1.1 appears as the natural choice. (Note that $F^{\prime}$ is full and faithful iff $\Phi^{\prime}$ is an isomorphism.)

Lemma 1.3. 1. Let $A \in \mathfrak{M}_{R}$. The $\operatorname{map} \Phi^{\prime}(A): \operatorname{Hom}(M, A) \bigotimes_{S} M \rightarrow$ $T_{M}(A)$ is an isomorphism if $M$ generates all kernels of homomorphisms $M^{n} \rightarrow A, n \in N$.

2. [17] The left $S$-module ${ }_{s} M$ is flat iff $M$ generates all kernels of homomorphisms $M^{n} \rightarrow M, n \in N$.

Proof. 1. Let $\sum_{i=1}^{n} f_{i}\left(m_{i}\right)=0$, where $f_{i} \in \operatorname{Hom}_{R}(M, A), m_{i} \in M$. By hypothesis $\left(m_{i}\right)_{1 \leqq i \leqq n}=\sum_{j=1}^{e}, g_{j}\left(n_{j}\right)$ for some $g_{i} \in \operatorname{Hom}_{R}\left(M, \operatorname{Ke}\left(\oplus f_{i}\right)\right)$, $n_{j} \in M$. Denoting the canonical projections $M^{n} \rightarrow M$ by $p r_{i}$, we conclude that $\sum_{i} f_{i} \otimes m_{i}=\sum_{i} f_{i} \otimes p r_{i}\left(\sum_{j} g_{j}\left(n_{j}\right)\right)=\sum_{i, j} f_{i} p r_{i} g_{j} \otimes n_{j}=0$, since $\sum_{i} f_{i} p r_{i} g_{j}=0$ for all $j$.

Assertion 2 is simply Lemma 19.19 of [2]. 
LEMMA 1.4. The following statements are equivalent.

(1) $M$ is a $\Sigma$-self-generator,

(2) $\mathscr{M}$ is closed with respect to $R$-submodules (hence is a Grothendieck category),

(3) $F^{\prime}$ is full and faithful, and $G^{\prime}$ is exact.

Proof. $\quad(1) \Leftrightarrow(2)$ : One direction is clear. Conversely, let $M$ be a $\Sigma$-self-generator, $A \in \mathscr{M}$ (i.e. there is a set $I$ and an epimorphism $f: M^{(I)} \rightarrow A$ ) and $A^{\prime}$ an $R$-submodule of $A$. Because $A^{\prime}$ may be assumed finitely generated, we can choose a finite subset $I^{\prime}$ of $I$ such that $A^{\prime} \subset f\left(M^{\left(I^{\prime}\right)}\right)$. By hypothesis, $M$ generates $f^{-1}\left(A^{\prime}\right) \cap M^{\left(I^{\prime}\right)}$ and hence $A^{\prime}$.

$(2) \Rightarrow(3)$ : This is a special case of [5] since $M$ is a generator for $\mathscr{L}$. It also follows directly from Lemma 1.3.

$(3) \Rightarrow(1)$ : Assume that $G^{\prime}$ is exact (i.e., ${ }_{s} M$ is flat). We claim that if $\Phi^{\prime}(A)$ is an isomorphism, then $M$ generates all kernels of homomorphisms $M^{n} \rightarrow A$ for $n \in N$. Let $f: M^{n} \rightarrow A$, let $f_{i}=f i n_{i}$ where $i n_{i}$ is the natural injection, and let $\left(m_{i}\right) \in \operatorname{Ke}(f)$. Assuming that $\Phi^{\prime}(A)$ is an isomorphism, $\Sigma f_{i}\left(m_{i}\right)=0$ forces the element $\Sigma f_{i} \otimes m_{i}$ of $\operatorname{Hom}(M, A) \otimes_{S} M$ to be zero. Consequently, since $s^{M}$ is flat, the element $\Sigma f_{i} \otimes m_{i}$ of $\Sigma f_{i} S \otimes M$ is zero. Thus, [3, Lemma 10] there are $g_{i j} \in S, n_{j} \in M, 1 \leqq i \leqq n, 1 \leqq j \leqq m$ such that

$$
\begin{array}{cr}
m_{i}=\sum_{j} g_{i j}\left(n_{j}\right), & \text { for all } i \\
\sum_{i} f_{i} g_{i j}=0, & \text { for all } j .
\end{array}
$$

For $g_{j}=\Sigma i n_{i} g_{i j}$, this means $g_{j} \in \operatorname{Hom}_{R}(M, \operatorname{Ke}(f))$ and $\left(m_{i}\right)=\sum_{j} g_{j}\left(n_{j}\right)$.

Remarks. 1. The implications $(3) \Rightarrow(2)$ and $(3) \Rightarrow(1)$ are independent of [5] where only Grothendieck categories are considered.

2. In our proof of $(3) \Rightarrow(1)$ we have shown that if ${ }_{s} M$ is flat, then the converse of Lemma 1.3.1 is true.

In part (1) of the following corollary we rediscover a theorem of Pahl [12] as the special case $A=M$ a generator.

Corollary 1.5. Let $M$ be a $\Sigma$-self-generator, $f: A \rightarrow B$ a homomorphism of $R$-modules. Then

1. $\operatorname{Hom}(M, A)$ is an injective (quasi-injective, see [2]) S-module iff $T_{M}(A)$ is an $M$-injective (quasi-injective) $R$-module.

2. $\operatorname{Hom}(M, f): \operatorname{Hom}(M, A) \rightarrow \operatorname{Hom}(M, B)$ is an essential monomorphism in $\mathfrak{M}_{S}$ iff $\left.f\right|_{T_{M^{(}(A)}}: T_{M}(A) \rightarrow T_{M}(B)$ is an essential monomorphism in $\mathfrak{M}_{R}$.

In particular, $F$ preserves injective envelopes.

3. If $\operatorname{Hom}(M, A)$ is artinian (noetherian) in $\mathfrak{M}_{s}$, then so is 
$T_{M}(A)$ in $\mathfrak{M}_{R}$ (e.g. if $S_{S}$ is artinian, then so is $M_{R}$ ).

Proof. 1.2. From 1.4. $\left(G^{\prime}, F^{\prime}\right)$ is an adjoint pair of functors between abelian categories, $G^{\prime}$ exact, $F^{\prime \prime}$ full and faithful. As is well-known, $F^{\prime}$ then preserves and reflects injectivity and essential extensions. Along the same line, one checks that $F^{\prime \prime}$ (hence $F$ ) preserves and reflects quasi-injectivity.

3. $A^{\prime} \mapsto \operatorname{Hom}_{R}\left(M, A^{\prime}\right)$ defines an injective map from the lattice of $R$-submodules of $T_{M}(A)$ into the lattice of $S$-submodules of $\operatorname{Hom}_{R}(M, A)$.

2. Perfect coinjectors. We call $M$ a (perfect) coinjector iff $F=\operatorname{Hom}_{R}(M,-): \mathfrak{M}_{R} \rightarrow \mathfrak{M}_{S}$ preserves injective modules (injective envelopes). It is well-known that $M$ is a coinjector iff $M$ is flat as a left $S$-module. As we have seen, all $\Sigma$-self-generators are perfect coinjectors. We will study cases, in which this is reversible and " $\Sigma$-self-generator" may be replaced by "self-generator". First of all, the special case of $R$ a Dedekind domain yields a structure theorem for perfect coinjectors. A resulting description of the torsion modules that are coinjectors generalizes [13, Th. 2]. For $0 \neq P \in \operatorname{Spec} R$, let $M_{P}=\left\{x \in M: P^{n} x=0\right.$ for some $\left.n \in N\right\}$.

THEOREM 2.1. For a Dedekind domain $R$, the following are equivalent:

(1) $M$ is a perfect coinjector,

(2) $F: \mathfrak{M}_{R} \rightarrow \mathfrak{M}_{S}$ preserves essential extensions,

(3) $M$ is a $\Sigma$-self-generator,

(4) $M$ is a self-generator,

(5) if $M$ is not a torsion module, then $M$ is a generator. If $M$ is a torsion module, then the following holds for each primary component $M_{P}, 0 \neq P \in$ Spec $R: M_{P}$ is reduced (i.e. does not contain a nonzero divisible submodule), or the direct complements of the largest divisible submodule are unbounded.

Furthermore, a torsion module is a perfect coinjector iff it is a coinjector.

Proof. (1) $\Rightarrow(2)$ and $(3) \Rightarrow(4)$ are trivial, $(3) \Rightarrow$ (1) holds for an arbitrary ring $R$. So does $(4) \Rightarrow(2)$ : Let $A \subset B$ be an essential extension and $0 \neq f \in \operatorname{Hom}_{R}(M, B)$. Pick $m \in M$ with $f(m) \neq 0$ and use the fact that $m R$ is generated by $M$ to find $g \in \operatorname{Hom}(M, m R) \subset S$ with $0 \neq f g \in \operatorname{Hom}_{R}(M, A)$.

$(2) \Rightarrow(5)$ : First, let $M$ be non-torsion, i.e. $R_{R}$ a submodule of $M_{R}$. The field $K$ of quotients of $R$ being an injective envelope of $R$ (as an $R$-module), $K_{R}$ is a direct summand of an injective envelope 
of $M$. This forces $\operatorname{Hom}_{R}(M, K) \neq 0$ and hence $\operatorname{Hom}_{R}(M, R) \neq 0$ by hypothesis. But for a Dedekind integral domain $R, T \neq 0$ means $T=R$.

For $M$ a torsion module, we may assume $M P$-primary and not reduced. Since $\bigcap_{n \in N} P^{n} M$ contains the largest divisible submodule of $M$, it is enough to prove $M / \bigcap_{n \in N} P^{n} M$ to be unbounded. Assume the contrary, $P^{k}\left(M / \bigcap_{n \in N} P^{n} M\right)=0$ for some $k \in N$. For $r \in P^{k} \backslash P^{k+1}$, let $l_{r}: M \rightarrow M$ be multiplication with $r$. By applying (2) to the essential extension $N \subset M$, where $N=\{m \in M: P m=0\}$, one obtains $g \in S$ such that $0 \neq l_{r} g \in \operatorname{Hom}_{R}(M, N)\left(l_{r} \neq 0\right.$, since $M$ is not reduced and hence unbounded). In particular, this means $P^{k+1} g(M)=0$. Consequently,

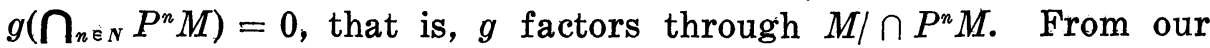
assumption we conclude $P^{k} g(M)=0$, contradicting $r g(M) \neq 0$.

$(5) \Rightarrow(3)$ : We limit our attention to a primary torsion module $M=M_{P}$ and a cyclic $R$-submodule $A \cong R / P^{k}$ of $M^{n}, n \in N$. If $M$ is bounded, then $M$ is known to be a direct sum of cyclic $R$-submodules, and so $A \subset M^{n}$ implies the existence of a direct summand $R / P^{n}$, where $n \geqq k$.

If $M$ is unbounded with $M_{1}$ its largest divisible submodule, then $M / M_{1}$ is also unbounded (in the case $M_{1} \neq 0$ apply the hypothesis). There is no loss of generality in assuming $M_{1}=0$, since $M_{1}$ is a direct summand of $M$. We claim $M / \bigcap_{n \in N} P^{n} M$ is unbounded. If not, then $P^{m} M \subset \bigcap_{n \in N} P^{n} M$ for some $m$ which would imply $P^{m} M=P^{m+i} M$, for all $i$. This means $P^{m} M$ is divisible. But with $M$ unbounded, $P^{m} M \neq 0$ contradicts $M_{1}=0$. In particular, we have that $P^{k}\left(M / P^{m} M\right) \neq 0$ for some $m$, and hence the bounded module $M / P^{m} M$ contains a direct summand of the form $R / P^{n}, n \geqq k$. Therefore, $M / P^{m} M$ resp. $M$ generates $A$. This completes the proof of the equivalences.

Now suppose a torsion module $M$ to be a coinjector. Lemma 1.3 justifies the restriction $M=M_{P}$. In order to verify (5), let $M=$ $M_{1} \oplus M_{2}$ with $0 \neq M_{1}$ divisible and $M_{2}$ reduced, $n \in N$ arbitrary. For $r \in P^{n}$, consider the multiplication $l_{r}: M \rightarrow M$ with $r$. From $1.3 \operatorname{ker}\left(l_{r}\right)$ is generated by $M$ and thus by $M_{2}$, because $\operatorname{Hom}\left(M_{1}, \operatorname{ker}\left(l_{r}\right)\right)=0$. Moreover, $\operatorname{ker}\left(l_{r}\right)$ contains a submodule $R / P^{n}$, since $M_{1}$ does, which forces $P^{n} M_{2} \neq 0$. This shows the unboundedness of $M_{2}$.

REMARKs. 1. The last statement is false for nontorsion modules: Consider the $\boldsymbol{Z}$-module $\boldsymbol{Q}$.

2. A different reading of $(4) \Leftrightarrow(5)$ for the special case $R=Z$ and $M$ a torsion module is [8, th 2.5].

3. Our proof actually establishes the implication $(4) \Rightarrow(2)$ for all rings $R$.

THEOREM 2.2. Let $R=\boldsymbol{Z}$. 
1. Direct sums of Prüfer groups $Z\left(p^{\infty}\right), p$ prime, are not coinjectors.

2. Direct sums of cyclic groups, especially all finitely generated or bounded groups, are perfect coinjectors.

3. A direct product of cyclic groups is a perfect coinjector iff it is either bounded or one of the cyclic factors is infinite.

Proof. 1., 2. are clear, 3. is left as an exercise.

Let $R$ be arbitrary. Example 1.2 shows that, in general, neither (2) implies (1) nor (4) implies (3). In the following we point out classes of modules $M$, for which the equivalence of the first four statements of 2.1 is maintained.

For an ideal $I$ of $R$, Sandomierski calls an $R$-module $A I$-accessible in case $A I=A$. With this definition, $M$ is $T$-accessible ("traceaccessible") if, for instance, $M$ is a projective module, a generator or an idempotent ideal (for further examples see $§ 3$ ).

LEMMA 2.3. (a) $M T=M$ iff $\Delta M=M$ iff $T_{M}(A)=A T$, for all $A \in \mathfrak{M}_{R}$.

(b) If $M T=M$, then $T$ and $\Delta$ are idempotent (i.e. $T^{2}=T, \Delta^{2}=\Delta$ ), and $\Delta$ is the trace of the left $S$-module $M$ (i.e. $\Delta=\Sigma\{\operatorname{Im}(g): g \in$ $\left.\left.\operatorname{Hom}_{S}(M, S)\right\}\right)$.

Proof. In view of $m(f, n)=[m, f] n$, a) and the first part of b) are straightforward. $\Delta \subset \Sigma\left\{\operatorname{Im}(g): g \in \operatorname{Hom}_{S}(M, S)\right\}$ is always true, because $[-, f] \in \operatorname{Hom}_{S}(M, S)$ for $f \in M^{*}$. The other inclusion follows from $\Delta M=M$.

THEOREM 2.4. For a module $M=M T$ or a quasi-projective module $M$ (see [2]), the following are equivalent:

(1) $M$ is a perfect coinjector,

(2) $F: \mathfrak{M}_{R} \rightarrow \mathfrak{M}_{S}$ preserves essential extensions,

(3) $M$ is a $\Sigma$-self-generator,

(4) $M$ is a self-generator,

(5) If $A^{\prime}$ is a simple essential submodule of an $R$-module $A$, then $\operatorname{Hom}\left(M, A^{\prime}\right)=0$ implies $\operatorname{Hom}(M, A)=0$,

In the case $M=M T$, we may add:

(6) $T_{R}$ is a self-generator,

(7) $R$-submodules of T-accessible modules are T-accessible,

(8) ${ }_{R}(R / T)$ is flat.

Remarks. 1. For $M$ projective, $(7) \Leftrightarrow(8)$ was proved independently in [10, Th. 2.1]. 
2. The proof of $(5) \Rightarrow(3)$ was inspired by [1, Th. 2.4] which is contained in the above as a special case of $(1) \Leftrightarrow(2) \Leftrightarrow(8)$. (Note that $M \otimes_{R}-\cong \operatorname{Hom}_{R}\left(M^{*},-\right)$, and ${ }_{R} M^{*}$ is finitely generated projective in case $M_{R}$ is finitely generated projective.)

3. $M$ quasi-projective and $M=M T$ are special cases of the following situation: There exists an $M$-projective module $P$ such that $M=\Sigma\{\operatorname{Im}(f): f \in S, f$ can be factored through $P\}$. Modules of this type (as well as $\Sigma$-self-generator) are easily checked to satisfy the following two conditions, for all submodules $A, B$ of $M^{n}, n \in N$ :

(a) $T_{M}(A+B)=T_{M}(A)+T_{M}(B)$.

(b) $A \subset B$ and $T_{M}(B) \subset T_{M}(A)$ implies $T_{M}(B / A)=0$. More general than 2.4, we prove the equivalence of (1)-(5) for all modules $M$ with conditions (a), (b). (Note that they do not, in general, hold for abelian groups.)

4. For $M_{R}=Q_{Z}$ the statements (6)-(8) are true, whereas (1)-(5) are not.

Proof of 2.4. Without restrictions on $M$, we have established $(3) \Rightarrow(1)$ in 1.5 and $(4) \Rightarrow(2)$ in the proof of 2.1. Also for all $M$ the implications $(1) \Rightarrow(2) \Longrightarrow(5)$ are trivially true. We show $(5) \Rightarrow(3)$ : Assume $x \notin T_{M}(x R)$ for some $x \in M^{n}, n \in N$. Choose $A \subset M^{n}$ maximal with respect to $T_{M}(x R) \subset A, x \notin A$. Then $A+x R / A$ is simple and essential in $M^{n} / A$. From $T_{M}(A+x R)=T_{M}(A)+T_{M}(x R) \subset T_{M}(A)$ we conclude $T_{M}(A+x R / A)=0$ (compare Remark 3$)$, hence $0=T_{M}\left(M^{n} / A\right)=$ $M^{n} / A$ by (5). This contradicts $M^{n} / A \neq 0$.

Now specialize to $M=M T$. In view of 1.4 and $T_{M}(A)=A T$, for all $A \in \mathfrak{M}_{R}$, conditions (3) and (7) are identical. By replacing $M$ by $T=T^{2}$ in $(1) \Rightarrow(2)$, we obtain $(6) \Rightarrow(7) . \quad(6) \Leftrightarrow(8)$ is easily derived from [3, p. 33].

$M$ being a perfect coinjector as described in 2.1 and $2.4, \operatorname{Hom}(M,-)$ also reflects injectivity and injective envelopes in the sense of 1.5. For example, if $M=M T$ is a self-generator, then $S$ is right injective iff $M_{R}$ is quasi-injective iff $M_{R}$ is $T$-injective.

3. Examples of $\Sigma$-self-generators $M=M T$. Trivially, every generator is a trace-accessible $\Sigma$-self-generator. Examples that arise for special classes of rings are listed in.

THEOREM 3.1. Let $M=M T$ (in particular true for $M_{R}$ projective). Then $M_{R}, T_{R}$ and ${ }_{R} T$ are trace-accessible $\Sigma$-self-generators if either.

1. $R$ is regular,

2. $R$ is commutative, and $M$ is projective or finitely generated. 
Proof. 1. is clear (2.4). 2. If $M$ is projective, then $M$ is a self-generator from [6]. The assertion follows from 2.4. Let $M$ be finitely generated. In order to verify (5) of 2.4 , we regard an essential extension of $R$-modules $A^{\prime} \subset A$ and $0 \neq f \in \operatorname{Hom}_{R}(M, A)$. Since $f(M)$ is finitely generated, there exists $r \in R$ with $0 \neq f(M) r \subset A^{\prime}$. This means $0 \neq l_{r} f \in \operatorname{Hom}_{R}\left(M, A^{\prime}\right)$, where $l_{r} \in S$ denotes the multiplication with $r$.

Not every $T$-accessible module $M$ over a commutative ring is a self-generator. For example, let $R$ be the ring of all Cauchy-sequences in $\boldsymbol{Q}$ with componentwise multiplication and $M$ the ideal of zero sequences. We observe $M=T=T^{2}$, whereas $M$ is not a selfgenerator: Pick $a=\left(a_{i}\right) \in T$ with $a_{i} \neq 0$, for infinitely many $i \in N$. Clearly $a \notin a T$, which means $a \notin T_{M}(a R)$. For $R$ arbitrary, not even the finitely generated and projective modules are self-generators. Choose $R=\left(\begin{array}{cc}K & K \\ 0 & K\end{array}\right)$, where $K$ is a field, $M=\left(\begin{array}{cc}K & K \\ 0 & 0\end{array}\right), A=\left(\begin{array}{ll}0 & K \\ 0 & 0\end{array}\right) \subset M$ and check $\operatorname{Hom}_{R}(M, A)=0$. (Consequently $M$ is not a perfect coinjector. However, $M$ is a coinjector, since $S$ is a field; compare [1]).

On the other hand, looking at $M$ as a left $S$-module, we make the following simple observation that will turn out to be very useful in $\S 5$.

THEOREM 3.2. For $M$ a projective $R$-module, the left $S$-modules $M$ and $\Delta$ are trace-accessible $\Sigma$-self-generators.

Proof. The trace of ${ }_{s} M$ coincides with $\Delta$, and we have $\Delta M=M$ (2.3). In view of 2.4 it is enough to show $m \in \Delta m$, for all $m \in M$, which is an immediate consequence of the dual basis lemma.

Examples of modules having the considered properties on both sides simultaneously are provided by the Zelmanowitz regular modules [18] (i.e. for every $m \in M$, there is an $f \in M^{*}$ satisfying $m=m(f, m)$ ). Part 1 of the following theorem contains [10, Cor. 2.2].

THEOREM 3.3. If $M_{R}$ is (Zelmanowitz) regular, then the following modules are trace-accessible $\Sigma$-self-generators:

1. the $R$-modules $M_{R}, T_{R}$,

2. the S-modules ${ }_{s} M,{ }_{s} \Delta$.

Proof. For $m \in M, m=m(f, m)$ is a stronger version of $m \in m T$, which means $M=M T$ is a self-generator. So 1 clearly follows from 2.4. Moreover, $m(f, m)=[m, f] m$, where $[-, f] \in \operatorname{Hom}_{S}(M, S)$, shows that ${ }_{S} M$ is again regular, and the above argument may be reflected to the other side. 
For examples of (Zelmanowitz) regular modules, especially nonprojective ones, see [18].

4. Submodules of $A_{R}$ and $\operatorname{Hom}_{R}(M, A)_{S^{*}}$. The following remarks on modules $M=M T$ form the basis for more specialized results for (finitely generated) projective modules on one hand and ( $T$-accessible self-) generators on the other. Lemma 4.1 and the symmetry of 4.2 with respect to $T$ and $\Delta$ show the condition $M=M T$ to be natural.

LEMMA 4.1. $\quad M=M T$ is true iff for every $A \in \mathfrak{M}_{R}$ and every $S$-submodule $B$ of $\operatorname{Hom}_{R}(M, A)$, the $S$-submodule $B \Delta$ of $B$ is essential.

Proof. Let $M=M T, A, B$ as above, and $0 \neq f \in B$. Pick $m \in M$ with $f(m) \neq 0$. By hypothesis, $m=\Sigma m_{i}\left(f_{i}, n_{i}\right)$, which means $0 \neq f(m)=\Sigma f\left(m_{i}\left(f_{i}, n_{i}\right)\right)=\Sigma f\left[m_{i}, f_{i}\right] n_{i}$, whence $0 \neq f\left[m_{i}, f_{i}\right] \in B \Delta$ for some $i$.

Conversely, we conclude $\operatorname{Hom}_{R}(M, M / M T)=0$ from $\operatorname{Hom}_{R}(M$, $M / M T) \Delta=0$.

Notation. For $A \in \mathfrak{M}_{R}$, the lattice of $R$-submodules resp. $T$ accessible $R$-submodules of $A$ will be denoted by $\mathscr{U}_{R}(A)$ resp. $\mathscr{U}_{T}(A)$. For $B \in \mathfrak{M}_{S}, \mathscr{U}_{S}(B)$ and $\mathscr{U}_{A}(B)$ are defined similarly.

THEOREM 4.2. Let $M=M T$.

1. For every $A \in \mathfrak{M}_{R}$, the following are inverse lattice isomorphisms:

$$
\begin{aligned}
& \psi: \mathscr{U}_{T}(A) \ni X \longmapsto \operatorname{Hom}(M, X) \Delta \in \mathscr{U}_{\Delta}(\operatorname{Hom}(M, A)) \\
& \varphi: \mathscr{U}_{\Delta}(\operatorname{Hom}(M, A)) \ni Y \longmapsto \Sigma\{\operatorname{Im}(f): f \in Y\} \in \mathscr{U}_{T}(A)
\end{aligned}
$$

2. Statements (1)-(3) resp. $\left(1^{\prime}\right)-\left(3^{\prime}\right)$ are equivalent:
(1) $\mathscr{U}_{T}(A)=\mathscr{U}_{R}(A T)$,
$\left(1^{\prime}\right) \mathscr{U}_{\Delta}(B)=\mathscr{U}_{S}(B \Delta)$, for all $A \in \mathfrak{M}_{R}$ for all $B \in \mathfrak{M}_{S}$
(2) $T_{R}$ (or $M_{R}$ ) is a
(2') $\Delta_{S}$ is a self-generator self-generator
(3) ${ }_{R}(R / T)$ is flat
$\left(3^{\prime}\right){ }_{s}(S / \Delta)$ is flat

Proof. 2. follows immediately from 2.4 .

1. In view of $T^{2}=T$ and $\Delta^{2}=\Delta$, the maps $\psi$ and $\varphi$ are welldefined lattice homomorphisms. Moreover, $\Delta M=M$ implies $\operatorname{Im}(f)=$ $\Sigma\{\operatorname{Im}(g): g \in f \Delta\}$, for all $f \in \operatorname{Hom}_{R}(M, A)$. For $X \in \mathscr{U}_{T}(A), \varphi \psi(X)=$ $\Sigma\{\operatorname{Im}(f): f \in \operatorname{Hom}(M, X) \Delta\}=\Sigma\{\operatorname{Im}(f): f \in \operatorname{Hom}(M, X)\}=X T=X$. 
Now let $Y \in \mathscr{U}_{\Delta}(\operatorname{Hom}(M, A))$. We claim $\operatorname{Hom}_{R}(M, X) \Delta=Y$, where $X=\Sigma\{\operatorname{Im}(f): f \in Y\}$. One inclusion is obvious. Conversely, let $h \in$ $\operatorname{Hom}_{R}(M, X),[m, g] \in \Delta$. From $h(m)=\Sigma f_{i}\left(m_{i}\right), f_{i} \in Y, m_{i} \in M$, we conclude $h[m, g]=\Sigma f_{i}\left[m_{i}, g\right] \in Y \Delta=Y$, since $h[m, g] x=h(m(g, x))=$ $h(m)(g, x)=\Sigma f_{i}\left(m_{i}\right)(g, x)=\Sigma f_{i}\left(m_{i}(g, x)\right)=\Sigma f_{i}\left[m_{i}, g\right] x$, for all $x \in M$.

\section{REMARKS AND COROLLARIES 4.3.}

1. The lattice isomorphism in 4.2 may also be deduced from [11, Prop. 6]. (This is more complicated but reveals a more general aspect.) For $\Delta=S$ it coincides with the one established by Sandomierski [15]. The symmetric extremes $T=R$ and $\Delta=S$ even have a converse in the following sense:

$T=R$ iff $\left.\mathscr{U}_{R}(A) \ni X \mapsto \operatorname{Hom}(M, X) \Delta \in \mathscr{U}_{\Delta}(\operatorname{Hom}(M, A))\right)$ is an isomorphism for all $A \in \mathfrak{M}_{R}$.

$\Delta=S$ iff $\mathscr{U}_{T}(A) \ni X \mapsto \operatorname{Hom}(M, X) \in \mathscr{\mathscr { C }}_{S}(\operatorname{Hom}(M, A))$ is an isomorphism for all $A \in \mathfrak{M}_{R}$.

2. Let $M=M T$. We illustrate with a few examples, how chain conditions of $A_{R}$ and $\operatorname{Hom}(M, A)_{S}$ are related (for $\Delta=S$, see [15]):

(a) $A T_{R}$ is finitely generated iff $\operatorname{Hom}(M, A) \Delta_{S}$ is finitely generated. In particular, $M_{R}$ is finitely generated iff $\Delta_{S}$ is finitely generated.

(b) If $M_{R}$ is a self-generator and $\operatorname{Hom}(M, A)_{S}$ is artinian (noetherian), then $A T_{R}$ is artinian (noetherian). If $\Delta_{S}$ is a self-generator and $A_{R}$ is artinian (noetherian), then $\operatorname{Hom}(M, A) \Delta_{S}$ is artinian (noetherian). (An interesting case being $A=A T=M$.)

Proof. (a) Let $A T_{R}$ be finitely generated, $\left(B_{i}\right)_{i \in I}$ a chain of proper $S$-submodules of $\operatorname{Hom}(M, A) \Delta$ and consider the chain $\left(B_{i} \Delta\right)_{i \in I}$. According to 4.2 , there is a chain $\left(X_{i}\right)_{i \in I}$ of proper $R$-submodules of $A T$ with $\psi\left(X_{i}\right)=B_{i} \Delta . \quad \bigcup X_{i} \varsubsetneqq A T$ (by hypotheses) and $\bigcup X_{i} \in \mathscr{U}_{T}(A)$ yields $\mathrm{U}\left(B_{i} \Delta\right)=\mathrm{U} \psi\left(X_{i}\right)=\psi\left(\mathrm{U} X_{i}\right) \varsubsetneqq \operatorname{Hom}(M, A) \Delta$, hence $\mathrm{U} B_{i} \varsubsetneqq$ $\operatorname{Hom}(M, A) \Delta$. The converse is proved similarly,

(b) is obvious.

3. Examples of modules $M$ such that $\Delta_{S}$ is a selfgenerator (different from $\Delta=S$ ) are easily deduced from [16, Th. 3.5]. In fact, the following are equivalent:

(1) $R$ is right noetherian,

(2) $\Delta_{S}$ is a $\left(\Sigma\right.$-)self-generator, for all projective modules $M_{R}$,

(3) $\quad M_{S}^{*}=M^{*} \Delta_{S}$ is a ( $\Sigma$-)self-generator, for all projective modules $M_{R}$.

For $R$ commutative noetherian, combine with 3.1 and 3.2: If $M_{R}$ is projective, then all of the following modules are $\Sigma$-self-generators: $M_{R}, T_{R}, M_{R}^{*},{ }_{S} M, M_{S}^{*},{ }_{S} \Delta, \Delta_{S}$. Consequently, $\mathscr{U}_{R}\left(M_{R}\right) \cong \mathscr{U}_{S}\left(\Delta_{S}\right)$, $\mathscr{U}_{R}\left(T_{R}\right) \cong \mathscr{U}_{S}\left(M_{S}^{*}\right)$. 
Sandmierski [15] called an $R$-module $X T$-faithful iff $x T \neq 0$, for all $0 \neq x \in X$, and proved that, in case $M_{R}$ is finitely generated projective, $X T$-faithful, then finite Goldie dimension of $X_{R}$ is inherited by $\operatorname{Hom}(M, X)_{S}$. The latter remains true for reduced hypothesis on $M$ and is even reversible.

CoROLlary 4.4. Let $M=M T, n \in N$.

1. If $X$ is T-faithful, then $X_{R}$ has finite Goldie dimension $n$ iff $\operatorname{Hom}(M, X)_{s}$ has finite Goldie dimension $n$.

2. If $M$ is a self-generator, then, for all $X \in \mathfrak{M}_{R}, X T_{R}$ has finite Goldie dimension $n$ iff the same is true for $\operatorname{Hom}(M, X)_{s}$.

Proof. 1. Let $\bigoplus_{i \in I} X_{i}$ be a direct sum of non-trivial $R$-submodules $X_{i}$ of $X$. We conclude $X_{i} T \neq 0$ by hypothesis and apply $\psi: \psi\left(\Sigma X_{i} T\right)=$ $\Sigma \psi\left(X_{i} T\right)$, where $0 \neq \psi\left(X_{i} T\right)_{S} \subset \operatorname{Hom}(M, X)_{S}$. The sum $\Sigma \psi\left(X_{i} T\right)$ is direct: $\quad X_{i} T \cap \sum_{i \neq j} X_{j} T=0$ implies $0=\psi\left(\left(X_{i} T \cap \sum_{i \neq j} X_{j} T\right) T\right)=$ $\left(\psi\left(X_{i} T\right) \cap \sum_{i \neq j} \psi\left(X_{j} T\right)\right) \Delta$, whence $\psi\left(X_{i} T\right) \cap \sum_{i \neq j} \psi\left(X_{j} T\right)=0$ from 4.1.

The same method yields the converse.

2. If $M$ is a self-generator, then $X T$ is $T$-faithful for all $X$ (4.2).

The information about the socle of $\operatorname{Hom}_{R}(M, A)_{s}[\operatorname{So}(\operatorname{Hom}(M, A))]$, given in the next theorem, characterizes self-generators. In the case of vector spaces, we rediscover standard results ( $\Delta$ being the ideal of $M$-endomorphisms of finite rank). For non-trivial examples see §3. Even the computation of So $\left(S_{S}\right)$ for $M$ a generator (e.g. $R=Z$, $M=\boldsymbol{Z} \oplus \boldsymbol{Z}\left(p^{\infty}\right)$ ) may be considerably simplified by 4.5 . " $\subset$ "' means “essential $R$-(resp. $S$-) submodule".

THEOREM 4.5. Let $M=M T, A \in \mathfrak{M}_{R}, X$ an $R$-submodule of $A T$, $B$ an $S$-submodule of $\operatorname{Hom}(M, A)$. Then the following are equivalent (condition (2)-(4) are understood to hold for all $A, X, B)$.

(1) $M_{R}$ is a self-generator,

(2) $\operatorname{Hom}_{R}(M, X) \Delta_{S}$ is simple iff $X_{R}$ is simple,

(2') $B \Delta_{S}$ is simple iff $\Sigma\{\operatorname{Im}(f): f \in B\}_{R}$ is simple,

(3) $\operatorname{Hom}(M, X) \subset^{\prime} \operatorname{Hom}(M, A)$ iff $X \subset^{\prime} A T$,

( $\left.3^{\prime}\right) \quad B \subset^{\prime} \operatorname{Hom}(M, A)$ iff $\Sigma\{\operatorname{Im}(f): f \in B\} \subset^{\prime} A T$,

(4) $\operatorname{So}(\operatorname{Hom}(M, A))=\operatorname{Hom}(M$, So $(A)) \Delta$, and one of the following is true:

(a) So $(\operatorname{Hom}(M, A)) \subset^{\prime} \operatorname{Hom}(M, A)$ iff So $(A T) \subset^{\prime} A T$,

(b) So $(\operatorname{Hom}(M, A))$ is simple iff So $(A T)$ is simple,

(c) $\operatorname{So}(\operatorname{Hom}(M, A))=0$ iff $\operatorname{So}(A T)=0$.

Moreover, if (1) holds, then $S$ is semisimple artinian iff $M_{R}$ is 
finitely generated, projective and semisimple (this generalizes [18, Th. 4.8.]).

Proof. $(1) \Longrightarrow(2)$ : Let $X_{R}$ be simple. From $X=X T$ (4.2) we deduce $\mathscr{U}_{T}(X)=\{0, X\}$, which means $\mathscr{U}_{\Delta}(\operatorname{Hom}(M, X) \Delta)=\{0, \operatorname{Hom}(M$, $X) \Delta$ \}. Moreover, for any $S$-submodule $B \neq 0$ of $\operatorname{Hom}(M, A) \Delta$, we obtain $B \Delta \neq 0$ (4.1); that is $B=B \Delta=\operatorname{Hom}(M, X) \Delta$. Analogously check the other implication of (2) with the aid of 4.2 .

$(2) \Rightarrow(1)$ : We verify condition (5) of 2.4. Let $A^{\prime}$ be a simple essential $R$-submodule of $A$ and $\operatorname{Hom}(M, A) \neq 0$, i.e. $A T \neq 0$. Hence $A^{\prime} \subset A T$. By (2), Hom $\left(M, A^{\prime}\right) \Delta$ is a simple $S$-module; in particular $\operatorname{Hom}\left(M, A^{\prime}\right) \neq 0$.

$(1) \Rightarrow(4)$ : Let $\left(B_{i}\right)_{i \in I}$ be the simple $S$-submodules of Hom $(M, A)$. We observe $B_{i} \Delta=B_{i}$ and choose $X_{i} \in \mathscr{U}_{T}(A)$ with $\psi\left(X_{i}\right)=B_{i}$ according to 4.2. By $(1) \Leftrightarrow(2)$ the $X_{i}$ are the simple $R$-submodules of $A T$, and we obtain: $\operatorname{So}(\operatorname{Hom}(M, A))=\sum_{i \in I} B_{i}=\sum_{i \in I} \psi\left(X_{i}\right)=\psi\left(\sum_{i} X_{i}\right)=$ $\psi(\operatorname{So}(A T))=\psi(\operatorname{So}(A) \cdot T)=\operatorname{Hom}(M$, So $(A)) \Delta$.

(a), (b), (c) follow immediately from 4.2.

$(4) \Rightarrow(1): \quad A, A^{\prime}$ as in “ $(2) \Rightarrow(1)$ ”. From $A^{\prime}=\operatorname{So}(A)=\operatorname{So}(A T)$ we deduce $\operatorname{So}(\operatorname{Hom}(M, A))=\operatorname{Hom}\left(M, A^{\prime}\right) \Delta$. So $(A T)$ being a simple, essential submodule of $A T$, we conclude $\operatorname{So}(\operatorname{Hom}(M, A)) \neq 0$ from each of (a), (b), (c). Consequently, $\operatorname{Hom}\left(M, A^{\prime}\right) \neq 0$.

The remaining implications are proved along the same pattern. Moreover, we note: $S$ is semisimple artinian iff $S=\operatorname{So}(S)=(\operatorname{Hom}(M$, So $(M))) \Delta$ iff $S=\Delta$ and So $(M)=M$.

5. Perfect injectors (compare [1]). In [1, Th. 2.4] Anderson established the equivalence of the following statements for a finitely generated projective module $M_{R}$ :

(i ) $M \bigotimes_{R}-:{ }_{R} \mathfrak{M} \rightarrow{ }_{S} \mathfrak{M}$ preserves injective envelopes $\left(M_{R}\right.$ is a "perfect injector"),

(ii) $M \otimes_{R}-:{ }_{R} \mathfrak{M} \rightarrow{ }_{S} \mathfrak{M}$ preserves essential extensions,

(iii) $(R / T)_{R}$ is flat,

From 2.4 we may add one more equivalent condition:

(iv) ${ }_{R} T$ is a self-generator.

As we will see, the background of this result is a category equivalence between the full subcategories of ${ }_{R} \mathfrak{M}$ resp. ${ }_{S} \mathfrak{M}$ consisting of all $T$-resp. $\Delta$-accessible objects (these will be denoted by ${ }_{T} \mathscr{M}$ resp. $\triangle \mathscr{C C}$ ). This observation will enable us to discuss the validity of either (i) or (ii) (which are not necessarily equivalent when "finitely generated" is dropped) and other properties of the functor $M \otimes_{R}$-.

Throughout this section we let $M=M T$; consequently, $M \bigotimes_{R} A \in$ ${ }_{\triangle} \mathscr{M}$, for all $A \in \in_{R} \mathfrak{M}$, and $M \bigotimes_{R} T A \cong M \bigotimes_{R} A$ in case $M_{R}$ is flat. The following theorem contains a variant of the Morita theorems. 
THEOREM 5.1. The following statements are equivalent:

(1) ${ }_{T} \mathscr{C l}$ and $\mathscr{M}$ are closed with respect to $R$-resp. S-submodules, and $M \otimes_{R}-$ induces an equivalence ${ }_{T} \mathscr{C l} \rightarrow{ }_{\triangle} \mathscr{C l}$ with inverse $M^{*} \otimes_{S}-$ (especially $M_{R}$ is flat),

(2) ${ }_{R} T$ and ${ }_{S} \Delta$ are self-generators,

(3) $(R / T)_{R}$ and $(S / \Delta)_{S}$ are flat.

Before proving 5.1, we notice that, for a projective or a regular module $M_{R}$, the left $S$-module ${ }_{S} \Delta$ is always a self-generator (3.2 and 3.3), hence in both of these cases (1) is true iff ${ }_{R} T$ is a self-generator. The following technical device contains [16, p. 358, cor.].

LEMma 5.2. 1. If ${ }_{R} T$ (or $T_{R}$ ) is a self-generator, then $T \cong$ $M^{*} \otimes_{S} M$ as $R$-bimodules,

2. If ${ }_{s} \Delta\left(\right.$ or $\left.\Delta_{S}\right)$ is a self-generator, then $\Delta \cong M \bigotimes_{R} M^{*}$ as $S$ bimodules.

Proof. 1. Let ${ }_{R} T$ be a self-generator. We show that (,): $M^{*} \otimes_{S} M \rightarrow T$ is an isomorphism. First, since $T M^{*}=M^{*} \Delta$ and $\Delta M=M$, we have $T M^{*} \bigotimes_{S} M=M^{*} \bigotimes_{S} M \in_{T} \mathscr{M}$. Since ${ }_{T} \mathscr{C l}$ is closed with respect to $R$-submodules (2.4), it is sufficient to show $T \cdot \mathrm{ke}()=$,0 . Let $\Sigma\left(f_{i}, m_{i}\right)=0$ and $(g, n) \in T$; then $(g, n) \Sigma f_{i} \otimes m_{i}=$ $\Sigma\left(g\left[n, f_{i}\right]\right) \otimes m_{i}=\Sigma g \otimes\left(\left[n, f_{i}\right] m_{i}\right)=g \otimes n \Sigma\left(f_{i}, m_{i}\right)=0$. The rest follows by symmetry.

Proof of 5.1. All of 5.1 is covered by 2.4 except the fact that (2) forces the restricted functors $M \otimes_{R}-:{ }_{T} \mathscr{C} \rightarrow{ }_{\triangle} \mathscr{M}$ and $M^{*} \bigotimes_{S}-$ : ${ }_{\Delta} \mathscr{C l} \rightarrow{ }_{T} \mathscr{C l}$ to be inverse equivalences. Since the inclusions $T_{R} \hookrightarrow R_{R}$ and $\Delta_{S} \subset S_{S}$ are pure by (2), we know $T A \cong T \bigotimes_{R} A$ and $\Delta B \cong$ $\Delta \bigotimes_{S} B$, for all $A \in_{R} \mathfrak{M}, B \in{ }_{S} \mathfrak{M}$. Now let $A \in_{T} \mathscr{M}, B \in_{\triangle} \mathscr{M}$. By combining the above with 5.2 , we obtain:

$$
\begin{aligned}
& A=T A \cong T \bigotimes_{R} A \cong M^{*} \bigotimes_{S} M \bigotimes_{R} A \\
& B=\Delta B \cong \Delta \bigotimes_{S} B \cong M \bigotimes_{R} M^{*} \bigotimes_{S} B
\end{aligned}
$$

From properties of the restriced functor $M \otimes_{R}-:{ }_{T} \mathscr{C} \rightarrow \mathscr{M}$, we easily derive information about the functor $M \otimes_{R}-:{ }_{R} \mathfrak{M} \rightarrow{ }_{S} \mathfrak{M}$.

COROLlary 5.3. One of the conditions of 5.1 being satisfied (e.g $M_{R}$ projective and $(R / T)_{R}$ flat), the statements of each of the following pairs are equivalent, for all $A, B \in_{R} \mathfrak{M}, f \in \operatorname{Hom}_{R}(A, B)$ :

1. (i) $M \otimes f: M \otimes_{R} A \rightarrow M \otimes_{R} B$ is an (essential) monomorphism in ${ }_{s} \mathfrak{M}$. 
(ii) $\left.f\right|_{T A}: T A \rightarrow T B$ is an (essential) monomorphism in ${ }_{R}^{\mathfrak{M}}$ (In case $f$ is an essential monomorphism, (ii) is true.)

2. (i) ${ }_{s} M \otimes_{R} A$ is $\Delta$-injective (quasi-injective).

(ii) ${ }_{R} A$ is $T$-injective (quasi-injective).

3. (i) $M \otimes f: M \bigotimes_{R} A \rightarrow M \bigotimes_{R} B$ is a projective cover in ${ }_{s} \mathbb{M}$.

(ii) $\left.f\right|_{T_{A}}: T A \rightarrow T B$ is a projective cover in ${ }_{R} \mathfrak{M}$ (In contrast to injective envelopes, $T A \rightarrow T B$ is not necessarily a projective cover in ${ }_{T} \mathscr{M}$ if $A \rightarrow B$ is a projective cover in ${ }_{R} \mathfrak{M}$.)

4. (i) $M \bigotimes_{R} A$ is artinian (noetherian, finitely generated) in ${ }_{S} \mathfrak{M}$. (ii) $T A$ is artinian (noetherian, finitely generated) in ${ }_{s} \mathfrak{M}$.

5.4. Connection with Anderson's results. If ${ }_{s} \Delta$ is a self-generator, as is the case when $M_{R}$ is projective or regular, then the statements (ii), (iii), (iv) of the beginning of this section are equivalent (for (ii) $\Rightarrow$ (iii) Anderson's proof may be adopted). However, (ii) does not imply (i): Let $M$ be a vector-space over a field $R, \operatorname{dim} M_{R}=\infty$, then ${ }_{s} M \cong$ ${ }_{S} M \otimes_{R} R$ is not injective (see [14]).

For the special case $\Delta=S$, the equivalence (ii) $\Leftrightarrow$ (i) follows from 5.3 .

EXAMPLE 5.5. The following classes of modules $M_{R}$ have the properties listed in 5.1 and 5.3, e.g. $M \otimes_{R}-$ preserves essential extensions:

1. All projective modules over a commutative ring $R$. In particular, all finitely generated projective modules over a commutative ring are perfect injectors in the sense of [1].

2. The maximal regular ideal [7] of an arbitrary ring $R$, considered as a right (resp. left) $R$-module.

Proof. 1. According to $3.1_{R} T$ and ${ }_{S} \Delta$ are self-generators.

2. The maximal regular ideal is Zelmanowitz regular as a right and left $R$-module. Hence, all the modules ${ }_{R} T, T_{R},{ }_{s} \Delta, \Delta_{S}$ are selfgenerators by 3.3 .

\section{REFERENCES}

1. F. W. Anderson, Endomorphism rings of projective modules, Math. Z., 111 (1969), 322-332.

2. F. W. Anderson and K. R. Fuller, Rings and Categories of Modules, Graduate Texts in Mathematics, Springer-Verlag, 1973.

3. N. Bourbaki, Algèbre Commutative, Chap. I, Hermann, Paris, 1961.

4. K. R. Fuller, Density and equivalence, J. of Algebra, 29 (1974), 528-550.

5. P. Gabriel and N. Popescu, Caractérisation des catégories abéliennes avec générateurs et limites inductives exactes, C. R. Acad. Sc. Paris, 258 (1964), 4188-4190.

6. G. Hauger and W. Zimmermann, Lokalisierung, Vervollständigung von Ringen und Bikommutatoren von Moduln, Algebra-Bericht Nr. 18, Math. Inst. d. Univ. München (1974). 
7. I. Kaplansky, Fields and Rings, The University of Chicago Press, 1972.

8. W. Liebert, Charakterisierung der Endomorphismenringe beschränkter abelscher Gruppen, Math. Ann., 174 (1967), 217-232.

9. R. W. Miller, Endomorphism rings of finitely generated projective modules, Pacific J. Math., 47 (1973), 199-220.

10. R. W. Miller, TTF-classes and quasi-generators, Pac. J. Math., 51 (1974), 499-507. 11. B. J. Mueller, The quotient category of a Morita context, J. of Algebra, 28 (1974), 389-407.

12. P. Pahl, Quasi-Frobenius-Generatoren und verallgemeinerte Dualitätsfragen. Dissertation München, 1964.

13. F. Richman and E. A. Walker, Primary abelian groups as modules over their endomorphism rings, Math. Zeitschr., 89 (1965), 77-81.

14. F. L. Sandomierski, Some examples of right self-injective rings which are not left self-injective, Proc. Amer. Math. Soc. 26 (1970), 244-245.

15. F. L. Sandomierski, Modules over the endomorphism ring of a finitely generated projective module, Proc. Amer. Math. Soc., 31 (1972), 27-31.

16. E. R. Willard, Properties of projective generators, Math. Annalen, 158 (1965), 352-364.

17. T. Würfel, Über absolvt reine Ringe, Algebra-Bericht Nr. 4, Math. Inst. d. Univ. München, 1973.

18. J. M. Zelmanowitz, Regular Modules, Trans. Amer. Math. Soc., 163 (197), 341-355.

Received May 15, 1975.

TeChnische Universität MÜNChen 


\section{PACIFIC JOURNAL OF MATHEMATICS}

\section{EDITORS}

RICHARD ARENS (Managing Editor)

University of California

Los Angeles, California 90024

\author{
R. A. Beaumont \\ University of Washington \\ Seattle, Washington 98105
}

\section{J. DugundjI}

Department of Mathematics University of Southern California Los Angeles, California 90007

D. Gilbarg and J. Milgram

Stanford University

Stanford, California 94305

\section{ASSOCIATE EDITORS}
E. F. BECKENBACH
B. H. NeumanN
F. WOLF
K. YoshidA

\section{SUPPORTING INSTITUTIONS}

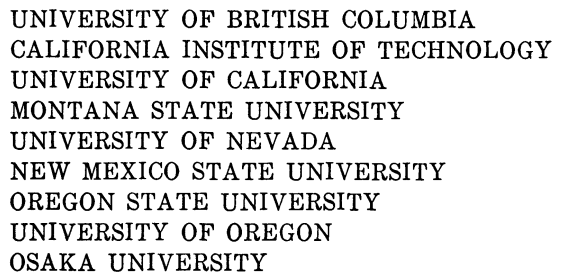

UNIVERSITY OF BRITISH COLUMBIA CALIFORNIA INSTITUTE OF TECHNOLOGY UNIVERSITY OF CALIFORNIA MONTANA STATE UNIVERSITY UNIVERSITY OF NEVADA NEW MEXICO STATE UNIVERSITY OSAKA UNIVERSITY OREGON STATE UNIVERSITY UNIVERSITY OF OREGON

\author{
UNIVERSITY OF SOUTHERN CALIFORNIA \\ STANFORD UNIVERSITY \\ UNIVERSITY OF TOKYO \\ UNIVERSITY OF UTAH \\ WASHINGTON STATE UNIVERSITY \\ UNIVERSITY OF WASHINGTON \\ AMERICAN MATHEMATICAL SOCIETY
}

The Supporting Institutions listed above contribute to the cost of publication of this Journal, but they are not owners or publishers and have no responsibility for its content or policies.

Mathematical papers intended for publication in the Pacific Journal of Mathematics should be in typed form or offset-reproduced, (not dittoed), double spaced with large margins. Please do not use built up fractions in the text of your manuscript. You may however, use them in the displayed equations. Underline Greek letters in red, German in green, and script in blue. The first paragraph or two must be capable of being used separately as a synopsis of the entire paper. Items of the bibliography should not be cited there unless absolutely necessary, in which case they must be identified by author and Journal, rather than by item number. Manuscripts, in triplicate, may be sent to any one of the editors. Please classify according to the scheme of Math. Reviews, Index to Vol. 39. All other communications should be addressed to the managing editor, or Elaine Barth, University of California, Los Angeles, California, 90024.

The Pacific Journal of Mathematics expects the author's institution to pay page charges, and reserves the right to delay publication for nonpayment of charges in case of financial emergency.

100 reprints are provided free for each article, only if page charges have been substantially paid. Additional copies may be obtained at cost in multiples of 50 .

The Pacific Journal of Mathematics is issued monthly as of January 1966. Regular subscription rate: $\$ 72.00$ a year $(6$ Vols., 12 issues). Special rate: $\$ 36.00$ a year to individual members of supporting institutions.

Subscriptions, orders for back numbers, and changes of address should be sent to Pacific Journal of Mathematics, 103 Highland Boulevard, Berkeley, California, 94708.

PUBLISHED BY PACIFIC JOURNAL OF MATHEMATICS, A NON-PROFIT CORPORATION

Printed at Kokusai Bunken Insatsusha (International Academic Printing Co., Ltd.), 8-8, 3-chome, Takadanobaba, Shinjuku-ku, Tokyo 160, Japan. 


\section{Pacific Journal of Mathematics}

\section{Vol. 61, No. 2 December, 1975}

Graham Donald Allen, Francis Joseph Narcowich and James Patrick Williams, An operator version of a theorem of Kolmogorov .......................

Joel Hilary Anderson and Ciprian Foias, Properties which normal operators share with normal derivations and related operators . . . . . . . . . . . . . . . . . . . . .

Constantin Gelu Apostol and Norberto Salinas, Nilpotent approximations and

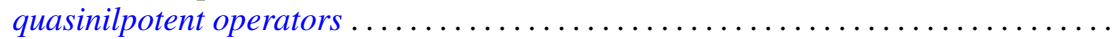

James M. Briggs, Jr., Finitely generated ideals in regular $F$-algebras . . . . . . . . . . .

Frank Benjamin Cannonito and Ronald Wallace Gatterdam, The word problem and power problem in 1-relator groups are primitive recursive ..................

Clifton Earle Corzatt, Permutation polynomials over the rational numbers ...........

L. S. Dube, An inversion of the $S_{2}$ transform for generalized functions . . . . . . . . . . William Richard Emerson, Averaging strongly subadditive set functions in unimodular

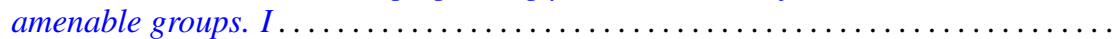

Barry J. Gardner, Semi-simple radical classes of algebras and attainability of

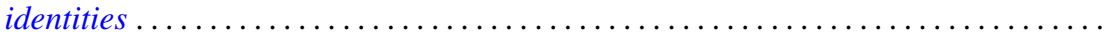

Irving Leonard Glicksberg, Removable discontinuities of A-holomorphic functions ....

Fred Halpern, Transfer theorems for topological structures . . . . . . . . . . . . . . .

H. B. Hamilton, T. E. Nordahl and Takayuki Tamura, Commutative cancellative

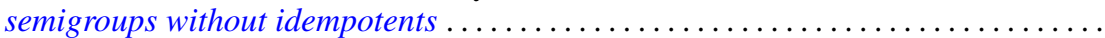

Melvin Hochster, An obstruction to lifting cyclic modules .....................

Alistair H. Lachlan, Theories with a finite number of models in an uncountable power

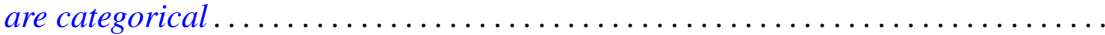

Kjeld Laursen, Continuity of linear maps from $C^{*}$-algebras . . . . . . . . . . . . .

Tsai Sheng Liu, Oscillation of even order differential equations with deviating arguments ....

Jorge Martinez, Doubling chains, singular elements and hyper- $Z$

Mehdi Radjabalipour and Heydar Radjavi, On the geometry of num Thomas I. Seidman, The solution of singular equations, I. Linear equations in Hilbert

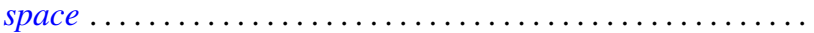

R. James Tomkins, Properties of martingale-like sequences ......

Alfons Van Daele, A Radon Nikodým theorem for weights on von Neumann

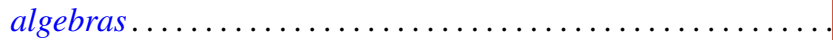

Kenneth S. Williams, On Euler's criterion for quintic nonresidues

Scott Andrew Wolpert, Noncompleteness of the Weil-Petersson metric for Teichmüller

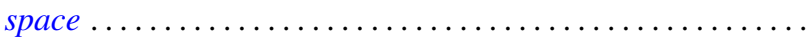

Volker Wrobel, Some generalizations of Schauder's theorem in locally convex

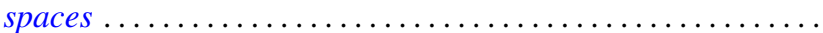

Kelly Denis McKennon, Corrections to: "Multipliers of type $(p, p)$ "; "Multipliers of type $(p, p)$ and multipliers of the group $L_{p}$-algebras"; "Multipliers and the

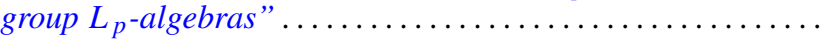

Andrew M. W. Glass, W. Charles (Wilbur) Holland Jr. and Stephen H. McCleary, Correction to: " $a *$-closures to completely distributive lattice-ordered

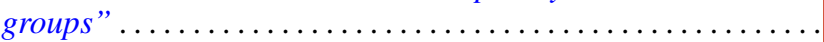

Zvi Arad and George Isaac Glauberman, Correction to: "A characteristic subgroup of

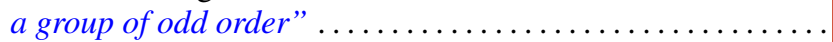

Roger W. Barnard and John Lawson Lewis, Correction to: "Subordination theorems

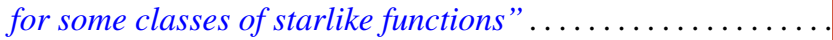

